Proyecciones Journal of Mathematics

Vol. 40, No 6, pp. 1569-1586, December 2021.

Universidad Católica del Norte

Antofagasta - Chile

\title{
Fixed point theorems in the study of positive strict set-contractions
}

\author{
Salima Mechrouk \\ Université de Boumerdes, Algeria \\ Received: August 2020. Accepted: June 2021
}

\begin{abstract}
The author uses fixed point index properties and Inspired by the work in Benmezai and Boucheneb (see Theorem 3.8 in [3]) to prove new fixed point theorems for strict set-contraction defined on a Banach space and leaving invariant a cone.
\end{abstract}

Keywords: Cones, fixed point theory, strict set-contractions, positive solution, general minorant principle, boundary value problem.

2010 Mathematics Subject Classifications: 47H10, 47H11, $47 H 30$. 


\section{Introduction}

In the study non-linear operators in ordered Banach spaces having an invariant cone it is often convenient to make use of minorants, majorants and the special concept of the derivatives in order to establish the existence of non-zero fixed points. Krasnoselskii has provided in [11] many interesting fixed point theorems stating that if such an operator is approximatively linear at 0 and $+\infty$, and the spectral radii of the linear approximations are oppositely located with respect to 1, then it has a fixed point. Amann in [2] has generalized these results for monotones operators which are strict set-contractions.

The main goal of this paper is to study strict-set contraction in ordered Banach spaces having an invariant cone and to give sufficient conditions on minorants and majorants which yield the existence of at least one non-zero fixed point ( see [4], [3], [1] and [5]). We will assume that the mapping $T$ has an asymptotically linear majorant $h$ and has a minorant $g$ which is right differentiable at zero and existence of the fixed point is obtained under additional conditions about the positive spectra of the derivatives. The proofs are based on the fixed point index theory, developed in [13] (see also the monographs [7] and [8]). In order to be more precise, let $X$ be a Banach space, $C$ be a cone in $X$, and let $T: C \longrightarrow C$ be a completely continuous mapping. Recently, Mechrouk have proved in [12] that if $T$ has a positive right differentiable at zero minorant $h: K \longrightarrow K$ and an asymptotically linear positive majorant $g: P \longrightarrow P$ satisfying $\theta_{P}^{g^{\prime}(\infty)}<1<\lambda_{P}^{h^{\prime}(0)}$, then $T$ has at least one positive nontrivial fixed point, where the constants $\lambda_{P}^{h^{\prime}(0)}$ and $\theta_{P}^{g^{\prime}(\infty)}$ play an important role in the statement of the obtained existence and nonexistence results and sometimes they replace the positive spectral radius. Motivated by the above work, we consider in this paper the case where the operator $T$ is a strict set-contraction.

The paper is organized as follows. Section 2 gives some preliminaries. Section 3 is devoted to prove new fixed point theorems for positive maps having approximative minorant and majorant at 0 and $\infty$ in specific classes of operators. Applications to the existence of solutions to a third order boundary value problem with mixed boundary conditions are presented in the last section. 


\section{Abstract Background}

We will use extensively in this work cones and the fixed point index theory, so let us recall some facts related to these two tools. Let $X$ be a real Banach space endowed with norm $\|$. $\|$, and let $L(X)=L(X, X)$ be the set of all linear continuous mapping from $X$ into $X$. A nonempty closed convex subset $C$ of $X$ is said to be a cone if $(t C) \subset C$ for all $t \geq 0$ and $C \cap(-C)=\left\{0_{X}\right\}$. It is well known that a cone $C$ induces a partial order in the Banach space $X$. We write for all $x, y \in X: x \preceq y$ if $y-x \in C, x \prec y$ if $y-x \in C, y \neq x$ and $x y$ if $y-x \notin C$. Notations $\succeq, \succ$ and denote respectively the reverse situations. We say that the cone $C$ is normal with a constant $n_{C}>0$ if for all $u, v$ in $C, u \preceq v$ implies $\|u\| \leq n_{C}\|v\|$.

Let $C$ be a cone in $X$ and let $L: X \rightarrow X$.

Definition 2.1. The mapping $L$ is said to be positive if $L(C) \subset C$. In this case, a nonnegative constant $\mu$ is said to be a positive eigenvalue of $L$ if there exists $u \in C\left\{0_{X}\right\}$ such that $L u=\mu u$.

Definition 2.2. Let $A$ be a nonempty set and let $B$ be an ordered set. $A$ map $g: A \longrightarrow B$ is said to be a majorant of the map $f: A \longrightarrow B$ if $f(x) \leq g(x)$ for all $x \in X$. Minorant is defined by reversing the above inequality sign.

Definition 2.3. Let $C$ be a cone in $X$ and $L: X \rightarrow X$ a continuous map.

$L$ is said to be

a) positive, if $L(C) \subset C$,

b) strongly positive, if $C$ has a nonempty interior (int $C \neq \emptyset)$ and $L\left(C\left\{0_{X}\right\}\right) \subset$ int $C$,

c) increasing, if for all $u, v \in X, u \preceq v$ implies $L u \preceq L v$.

Definition 2.4. Let $L_{1}, L_{2}: X \rightarrow X$ be positive maps. We write $L_{1} \preceq L_{2}$ if for all $x \in C, L_{1} x \preceq L_{2} x$.

Definition 2.5. Let $B(X)$ be the set of all bounded subsets of $X$ and $\psi: B(X) \longrightarrow \mathbf{R}^{+}$be a measure of non-compactness on $X$; that is $\psi$ satisfies for $A, B \in B(X)$

1. $\psi(A)=0 \Longleftrightarrow A$ is relatively compact on $X$.

2. $A \subseteq B$ imply $\psi(A) \leq \psi(B)$.

3. $\psi(\overline{c o} A)=\psi(\bar{A})=\psi(A)$. 
4. $\psi(A \cup B)=\max \{\psi(A), \psi(B)\}$.

5. for all $t \in[0,1], \psi(t A+(1-t) B) \leq t \psi(A)+(1-t) \psi(B)$

6. if $\left(A_{n}\right)_{n} \subset B(X)$ is a decreasing sequence of closed nonempty sets with $\lim \psi\left(A_{n}\right)=0$, then $\cap_{n \geq 1} A_{n}$ is a nonempty compact set.

Definition 2.6. A function $f: \Omega \subset X \rightarrow X$ is said to be a strict-set contraction if it is continuous, bounded, and there exists a constant $k \in$ $[0,1)$ such that $\psi(f(S)) \leq k \psi(S)$ for all bounded sets $S \subset \Omega$.

Definition 2.7 ([14]). A map $g: C \longrightarrow X$ is said to be differentiable at $x_{0} \in C$ along $C$ if there exists $g^{\prime}\left(x_{0}\right) \in L(X)$ such that

$$
\lim _{h \in C, h \longrightarrow 0} \frac{\left\|g\left(x_{0}+h\right)-g\left(x_{0}\right)-g^{\prime}\left(x_{0}\right) h\right\|}{\|h\|}=0 .
$$

We say that $g^{\prime}\left(x_{0}\right)$ is the derivative of $g$ at $x_{0}$ along $C$, is uniquely determined.

The map $g$ is said to be asymptotically linear along $C$ if there exists $g^{\prime}(\infty) \in L(X)$ such that

$$
\lim _{x \in C,\|x\| \longrightarrow+\infty} \frac{\left\|g(x)-g^{\prime}(\infty) x\right\|}{\|x\|}=0 .
$$

Again, $g^{\prime}(\infty)$ is uniquely determined and called the derivative at infinity along $C$.

Lemma 2.8 ([11]). The derivative $g^{\prime}(\nu),\left(\nu=+\infty\right.$, or $\left.x_{0} \in C\right)$, with respect to a cone of the positive operator $g$ is a linear positive operator.

Detailed presentation of the differentiability with respect to a cone can be found in [11] and [14].

The main results of this paper are proved by means of the fixed point index theory for strict-set contraction mappings develloped in [13].

Let us recall some lemmas providing fixed point index computations. Let $C$ be a cone in $X$. Let for $R>0, C_{R}=C \cap B\left(0_{X}, R\right)$ where $B\left(0_{X}, R\right)$ is the open ball of radius $R$ centred at $0_{X}$, and let $\partial C_{R}$ be its boundary and consider a strict-set contraction mapping, $f: \overline{C_{R}} \rightarrow C$. 
Lemma 2.9 ([7]). If $f x \neq \lambda x$ for all $x \in \partial C_{R}$ and $\lambda \geq 1$ then $i\left(f, C_{R}, C\right)=$ 1.

Lemma 2.10 ([7]). If there exists $e \succ 0_{X}$ such that $x \neq f x+$ te for all $t \geq 0$ and all $u \in \partial C_{R}$ then $i\left(f, C_{R}, C\right)=0$.

From the two Lemma above, we conclude the following Lemma.

Lemma 2.11. If $f x x$ for all $x \in \partial C_{R}$ then $i\left(f, C_{R}, C\right)=1$.

Lemma 2.12. If $f x x$ for all $x \in \partial C_{R}$ then $i\left(f, C_{R}, C\right)=0$.

A detailed presentation of the fixed point index theory for strict-set contraction mappings can be found in [13].

In all this section $E$ is a real Banach space, $K$ is a nontrivial cone in $E$ and $L(E)$ denote the set of all linear continuous self mapping on $E$ endowed with the norm, $\|L\|=\sup _{\|u\|=1}\|L u\|$. Let $C^{+}(E)$ denote the subset of $L(E)$ consisting of all strict set-contraction positive operators. Hereafter $\preceq$ is the order induced by the cone $K$ on $E$ and we set,

$$
L_{K}(E)=\{L \in L(E), L \text { is increasing }\}
$$

and

$$
C_{K}(E)=\left\{L \in L_{K}(E): L \text { is a strict-set contraction }\right\} .
$$

Now, for $L \in L_{K}(E)$ we define the subset

$$
\Theta_{P}^{L}=\left\{\theta \geq 0 \text { : there exists } u \in P\left\{0_{E}\right\} \text { such that } L u \succeq \theta u\right\} .
$$

Remark 2.13. Note that

i) $0 \in \Theta_{P}^{L}$ and if $\theta \in \Theta_{P}^{L}$, then $[0, \theta] \subset \Theta_{P}^{L}$.

ii) $\Lambda_{P}^{L} \subset \Lambda_{K}^{L}$ and $\Theta_{P}^{L} \subset \Theta_{K}^{L}$.

iii) If $\mu$ is positive eigenvalue of $L$, then $\mu \in \Theta_{P}^{L} \cap[0,\|L\|]$.

iv) If $L^{-1}\left(0_{E}\right) \cap K=\left\{0_{E}\right\}$ and $P \subset K$ then $\Theta_{P}^{L}=\Theta_{K}^{L}$.

In all this paper, we set for $L \in L_{K}^{P}(E)$,

$$
\theta_{P}^{L}=\sup \Theta_{P}^{L}
$$

The constant $\theta_{P}^{L}$ replaces the spectral radius of $L$ which in our case is not necessarily an eigenvalue of $L$ having an eigenvector in $K$. So, it is 
natural to ask what represents this constant with respect to the operator $L$.

If $L: E \longrightarrow E$ is a bounded linear operator, then we define, $r(L)$, its spectral radius by

$$
r(L)=\lim _{n \longrightarrow+\infty}\left\|L^{n}\right\|^{\frac{1}{n}} .
$$

Lemma 2.14 gives sufficient conditions for the existence of $\theta_{P}^{L}$.

Lemma 2.14 ([3]). Assume $L \in L_{K}(E)$. Then the subset $\Theta_{P}^{L}$ is bounded from above by $r(L)$.

Lemma 2.15 ([3]). Assume that the cone $K$ is solid, and let $L \in C_{K}(E)$ be strongly positive and increasing. Then $\theta_{K}^{L}$ is the unique positive eigenvalue of $L$.

Lemma 2.16 ([10]). Let $D \subset E, D$ be a bounded set and $f$ is uniformly continuous and bounded from $J \times S$ into $E$, then

$$
\psi(f(J \times S))=\max _{t \in J} \psi(f(t, S), \forall S \subset D .
$$

\section{Main results}

Lemma 3.1. Suppose that $T$ has a right differentiable at zero majorant $g: K \longrightarrow K$ such that $g(0)=0, g^{\prime}(0) \in C_{K}(E)$ satisfying $r\left(g^{\prime}(0)\right)<1$ and $K$ is a normal cone. Then $T$ has at least one positive fixed point.

Proof. Let us prove existence of $r>0$ small enough, such that for all $t \in[0,1]$ equation $t T u=u$ has no solution in $\partial K_{r}=K \cap B\left(0_{E}, r\right)$ where $B\left(0_{E}, r\right)$ is the open ball of radius $r$ centred at $0_{E}$, and let $\partial K_{r}$ be its boundary.

By the contrary suppose that for each integer $n \geq 1$ there exist $t_{n} \in[0,1]$ and $u_{n} \in \partial K_{\frac{1}{n}}$, such that

$$
u_{n}=t_{n} T u_{n}
$$

Note that $v_{n}=u_{n} /\left\|u_{n}\right\| \in \partial K_{1}$ and satisfies

$$
v_{n} \preceq \frac{g\left(u_{n}\right)}{\left\|u_{n}\right\|} .
$$

Thus, we have: 


$$
\frac{g\left(u_{n}\right)}{\left\|u_{n}\right\|}=\frac{g\left(u_{n}\right)-g^{\prime}(0)\left(u_{n}\right)}{\left\|u_{n}\right\|}+\frac{g^{\prime}(0)\left(u_{n}\right)}{\left\|u_{n}\right\|} .
$$

We set

$$
G_{n}\left(u_{n}\right)=\frac{g\left(u_{n}\right)-g^{\prime}(0)\left(u_{n}\right)}{\left\|u_{n}\right\|} .
$$

Clearly

$$
v_{n} \preceq G_{n}\left(u_{n}\right)+g^{\prime}(0)\left(v_{n}\right) .
$$

We obtain from (3.3), that is

$$
\begin{aligned}
v_{n} & \preceq G_{n}\left(u_{n}\right)+g^{\prime}(0)\left(v_{n}\right) \\
& \preceq G_{n}\left(u_{n}\right)+g^{\prime}(0)\left(G_{n}\left(u_{n}\right)\right)+\left[g^{\prime}(0)\right]^{2}\left(v_{n}\right) \\
& \preceq G_{n}\left(u_{n}\right)+g^{\prime}(0)\left(G_{n}\left(u_{n}\right)\right)+\left[g^{\prime}(0)\right]^{2}\left(G_{n}\left(u_{n}\right)\right)+\left[g^{\prime}(0)\right]^{3}\left(v_{n}\right) \\
& \vdots \\
& \preceq I_{n, k}+\left[g^{\prime}(0)\right]^{k}\left(v_{n}\right)
\end{aligned}
$$

where $I_{n, k}=\sum_{i=0}^{k}\left[g^{\prime}(0)\right]^{i}\left(G_{n}\left(u_{n}\right)\right)$ and we have from the normality of the cone $K$

$$
\begin{aligned}
1 & \leq c_{K}\left\|I_{n, k}\right\|+c_{K}\left\|\left[g^{\prime}(0)\right]^{k}\left(v_{n}\right)\right\| \\
& \leq c_{K}\left\|I_{n, k}\right\|+c_{K}\left\|\left[g^{\prime}(0)\right]^{k}\right\|
\end{aligned}
$$

in which by letting $n \longrightarrow \infty$, yields $1 \leq c_{K}\left\|\left[g^{\prime}(0)\right]^{k}\right\|$. Then $1 \leq c_{K}^{\frac{1}{k}} \|$ $\left[g^{\prime}(0)\right]^{k} \|^{\frac{1}{k}}$. leading to the contradiction by letting $k \longrightarrow \infty, 1 \leq r\left(g^{\prime}(0)\right)<$ 1 , and proves existence of $r>0$ small enough such that for all $t \in[0,1]$ equation $t T u=u$ has no solution in $\partial K_{r}$. For a such $r>0$, we deduce from Lemma 2.9 that

$$
i\left(T, K_{r}, K\right)=1
$$

and $T$ has a positive fixed point $u$ with $\|u\|<r$. This completes the proof.

Arguing as in the proof of Theorem 3.1, we obtain the following result.

Lemma 3.2. Suppose that $T$ has an asymptotically linear majorant $g$ : $K \longrightarrow K$ such that $g^{\prime}(\infty) \in C_{K}(E)$ satisfying $r\left(g^{\prime}(\infty)\right)<1$ and $K$ is normal. Then $T$ has at least one positive fixed point. 
Theorem 3.3. Suppose that the cone $K$ is a normal cone and $T$ has an asymptotically linear majorant $g: K \longrightarrow K$ such that $g^{\prime}(\infty) \in C_{K}(E)$. Suppose that $T$ has a right differentiable at zero minorant $h: K \longrightarrow K$ such that $h(0)=0$ and $h^{\prime}(0) \in C_{K}(E)$ satisfying $r\left(g^{\prime}(\infty)\right)<1<\theta_{P}^{h^{\prime}(0)}$. Then $T$ has at least one positive nontrivial fixed point.

Proof. We have to prove existence of $0<r<R$ such that

$$
i\left(T, K_{r}, K\right)=0 \text { and } i\left(T, K_{R}, K\right)=1 .
$$

In such a situation, additivity and solution properties of the fixed point index imply that

$$
i\left(T, K_{R} \overline{K_{r}}, K\right)=i\left(T, K_{R}, K\right)-i\left(T, K_{r}, K\right)=1
$$

and $T$ has a positive fixed point $u$ with $r<\|u\|<R$.

Let us prove existence of $R>0$ Big enough, such that for all $t \in[0,1]$ equation $t T u=u$ has no solution in $\partial K_{R}$. By the contrary suppose that for each integer $n \geq 1$ there exist $t_{n} \in[0,1]$ and $u_{n} \in \partial K_{n}$ such that

$$
u_{n}=t_{n} T u_{n}
$$

Note that $w_{n}=u_{n} /\left\|u_{n}\right\| \in \partial K_{1}$ and satisfies

$$
w_{n} \leq \frac{g\left(u_{n}\right)}{\left\|u_{n}\right\|} .
$$

Thus, we have:

$$
\frac{g\left(u_{n}\right)}{\left\|u_{n}\right\|}=\frac{g\left(u_{n}\right)-g^{\prime}(\infty)\left(u_{n}\right)}{\left\|u_{n}\right\|}+\frac{g^{\prime}(\infty)\left(u_{n}\right)}{\left\|u_{n}\right\|} .
$$

We set

$$
G_{n}\left(u_{n}\right)=\frac{g\left(u_{n}\right)-g^{\prime}(\infty)\left(u_{n}\right)}{\left\|u_{n}\right\|} .
$$

Clearly

$$
w_{n} \preceq G_{n}\left(u_{n}\right)+g^{\prime}(\infty)\left(w_{n}\right) .
$$

By the same argument used in Lemma 3.2, we obtain that:

$$
w_{n} \preceq J_{n, k}+\left[g^{\prime}(\infty)\right]^{k}\left(w_{n}\right) .
$$

where $J_{n, k}=\sum_{i=0}^{k}\left[g^{\prime}(\infty)\right]^{i}\left(G_{n}\left(u_{n}\right)\right)$ and we have from the normality of the cone $K$ 


$$
\begin{aligned}
1 & \leq c_{K}\left\|J_{n, k}\right\|+c_{K}\left\|\left[g^{\prime}(\infty)\right]^{k}\left(v_{n}\right)\right\| \\
& \leq c_{K}\left\|J_{n, k}\right\|+c_{K}\left\|\left[g^{\prime}(\infty)\right]^{k}\right\|
\end{aligned}
$$

in which by letting $n \longrightarrow \infty$, yields $1 \leq c_{K}\left\|\left[g^{\prime}(\infty)\right]^{k}\right\|$. Then $1 \leq$ $c_{K}^{\frac{1}{k}}\left\|\left[g^{\prime}(\infty)\right]^{k}\right\|^{\frac{1}{k}}$. leading to the contradiction by letting $k \longrightarrow \infty, 1 \leq$ $r\left(g^{\prime}(\infty)\right)<1$, and proves existence of $R>0$ big enough such that for all $t \in[0,1]$ equation $t T u=u$ has no solution in $\partial K_{R}$. For a such $R>0$, we deduce from Lemma 2.9 that

$$
i\left(T, K_{R}, K\right)=1
$$

Let $e>0$ such that $h^{\prime}(0)(e) \succeq \theta_{K}^{h^{\prime}(0)} e$ and let us prove existence of $r>0$ small enough, such that for all $t>0$ equation $T u+t e=u$ has no solution in $\partial K_{r}$. By the contrary suppose that for each integer $n \geq 1$ there exist $t_{n} \in \mathbf{R}^{+}$and $u_{n} \in \partial K_{\frac{1}{n}}$ such that

$$
u_{n}=T u_{n}+t_{n} e .
$$

Note that $v_{n}=u_{n} /\left\|u_{n}\right\| \in \partial K_{1}$ and satisfies the inequality:

$$
v_{n} \succeq\left(T u_{n} /\left\|u_{n}\right\|\right) \succeq \frac{h\left(u_{n}\right)}{\left\|u_{n}\right\|} .
$$

Thus, we have

$$
\frac{h\left(u_{n}\right)}{\left\|u_{n}\right\|}=\frac{h\left(u_{n}\right)-h^{\prime}(0)\left(u_{n}\right)}{\left\|u_{n}\right\|}+\frac{h^{\prime}(0)\left(u_{n}\right)}{\left\|u_{n}\right\|} .
$$

We set

$$
H_{n}\left(u_{n}\right)=\frac{h\left(u_{n}\right)-h^{\prime}(0)\left(u_{n}\right)}{\left\|u_{n}\right\|} .
$$

Then, one has

$$
v_{n} \succeq H_{n}\left(u_{n}\right)+h^{\prime}(0)\left(v_{n}\right) .
$$

and

$$
v_{n} \succeq \frac{t_{n} e}{\left\|u_{n}\right\|} \succeq t_{n} e
$$

We obtain 


$$
\begin{aligned}
v_{n} & \succeq H_{n}\left(u_{n}\right)+h^{\prime}(0)\left(v_{n}\right) \\
& \succeq H_{n}\left(u_{n}\right)+h^{\prime}(0)\left(H_{n}\left(u_{n}\right)\right)+\left[h^{\prime}(0)\right]^{2}\left(v_{n}\right) \\
& \succeq H_{n}\left(u_{n}\right)+h^{\prime}(0)\left(H_{n}\left(u_{n}\right)\right)+\left[h^{\prime}(0)\right]^{2}\left(H_{n}\left(u_{n}\right)\right)+\left[h^{\prime}(0)\right]^{3}\left(v_{n}\right) \\
& \vdots \\
& \succeq H_{n}\left(u_{n}\right)+h^{\prime}(0)\left(H_{n}\left(u_{n}\right)\right)+\left[h^{\prime}(0)\right]^{2}\left(H_{n}\left(u_{n}\right)\right)+\cdots+\left[h^{\prime}(0)\right]^{k}\left(H_{n}\left(u_{n}\right)\right)+\left[h^{\prime}(0)\right]^{k}\left(v_{n}\right) \\
& =I_{n, k}+\left[h^{\prime}(0)\right]^{k}\left(v_{n}\right) .
\end{aligned}
$$

We have from (3.9)

$$
v_{n} \succeq I_{n, k}+t_{n}\left(\theta_{K}^{h^{\prime}(0)}\right)^{k} e
$$

where $I_{n, k}=\sum_{i=0}^{k}\left[h^{\prime}(0)\right]^{i}\left(H_{n}\left(u_{n}\right)\right)$ and the normality of $K$ leads to

$$
c_{K}\left\|v_{n}\right\| \geq t_{n}\left(\theta_{K}^{h^{\prime}(0)}\right)^{k}\|e\|-\left\|I_{n, k}\right\|
$$

letting $n \rightarrow \infty$, we obtain

$$
c_{K} \geq t\left(\theta_{K}^{h^{\prime}(0)}\right)^{k}\|e\|
$$

Taking in account, $\theta_{K}^{h^{\prime}(0)}>1$, we obtain the contradiction

$$
0_{E} \prec t\|e\| \preceq c_{K} /\left(\theta_{K}^{h^{\prime}(0)}\right)^{k} \rightarrow 0_{E} \text { as } k \rightarrow \infty .
$$

Thus, we have from Lemma $2.10, i\left(T, K_{r}, K\right)=0$. This completes the proof.

\subsection{Application to second order bvp}

Throughout the remainder of this paper, we apply the above results to a second-order differential equation in Banach spaces:

$$
\begin{cases}u^{\prime \prime}(t)+f(t, u(t))=\theta & 0<t<1 \\ u(0)=\int_{0}^{1} s u(s) d s u(1)=\theta, & \end{cases}
$$


where $f \in C[[0,1] \times P, P, \theta$ is the zero element of $E$.

We consider problem $(3.11)$ in $C(J, E)$, with $J=[0,1]$. Clearly that $\left(C(J, E),\|\cdot\|_{c}\right)$ is a Banach space with the norm $\|u\|_{c}=\max _{t \in J}\|u(t)\|$ for $u \in C(J, E)$.

We suppose that:

(H) $f \in C[J \times P, P]$, and let $l>0, f(t, x)$ is uniformly continuous on $J \times\left(P \cap S_{l}\right)$ and there exists a constant $L_{l}$ with $0 \leq L_{l}<\frac{5}{2}$ such that

$$
\psi(f(t, S)) \leq L_{l} \psi(S), \forall t \in J, S \subset P \cap S_{l},
$$

where $S_{l}=\{u \in E,\|u\|<r\}$ and here $\psi$ denotes the Kuratowski measure of non-compactness on $S$.

Tt is easy to see that the problem (3.11) has a solution $u=u(t)$ if and only if $u$ is a solution of the operator equation

$$
T u(x)=\int_{0}^{1} H(t, s) f(s, u(s)) d s,
$$

where $H(t, s)$ is the Green's function associated with (3.11) given by

$$
H(t, s)=G(t, s)+\frac{6}{5}(1-t) \int_{0}^{1} \tau G(s, \tau) d \tau,
$$

where

$$
G(t, s)= \begin{cases}t(1-s) & t \leq s, \\ s(1-t) & s \leq t .\end{cases}
$$

Let $e(x)=x(1-x), \forall x \in[0,1]$. We may prove the following Lemma.

Lemma 3.4. The Green's function $G(t, s)$ possesses the following properties:

$1 G(t, s) \geq 0, \forall t, s \in[0,1]$.

$2 e(t) e(s) \leq G(t, s) \leq e(t) \leq \bar{e}=\max _{[0,1]} e(t)=\frac{1}{4}$.

$3 G(t, s) \leq e(s), \forall t, s \in[0,1]$.

4 Let $\delta \in\left(0, \frac{1}{2}\right), J_{\delta}=[\delta, 1-\delta]$, then

$$
G(t, s) \geq \delta G(\tau, s), \forall t \in J_{\delta} \forall \tau, s \in[0,1] .
$$

Proposition 3.5. Assume that $(H)$ hold. Then for $t, s \in[0,1]$, we have:

$$
\frac{1}{10} e(t) e(s) \leq H(t, s) \leq \frac{8}{5} e(s) \leq \frac{2}{5}=B_{H}, \forall t, s \in[0,1] .
$$




\section{Proof.}

$$
\begin{aligned}
H(t, s) & \geq \frac{6}{5}(1-t) \int_{0}^{1} \tau G(s, \tau) d z \\
& \geq \frac{6}{5}(1-t) \int_{0}^{1} \tau e(s) e(\tau) d z \\
& =\frac{6}{5} t(1-t) e(s) \int_{0}^{1} \tau e(\tau) d z \\
& =\frac{6}{5} \frac{1}{12} e(t) e(s) \\
& =\frac{1}{10} e(t) e(s) .
\end{aligned}
$$

In other hand, since $G(t, s) \leq e(s)$, we have:

$$
\begin{aligned}
H(t, s) & \leq G(t, s)+\frac{6}{5}(1-t) \int_{0}^{1} \tau e(s) d z \\
& \leq e(s)+\frac{6}{5} e(s) \int_{0}^{1} \tau e(s) e(\tau) d z \\
& =\frac{8}{5} e(s)
\end{aligned}
$$

Proposition 3.6. Assume that $(H)$ hold. Then for $t \in J_{\delta}, \tau, s \in[0,1]$, we have:

$$
H(t, s) \leq \delta H(\tau, s)
$$

\section{Proof.}

$$
\begin{aligned}
H(t, s) & \geq \delta G(\tau, s)+\frac{6}{5} \int_{0}^{1} G(s, \tau) \tau d z \\
& \geq \delta G(\tau, s)+\frac{6 \delta}{5} \int_{0}^{1} G(s, \tau) \tau d z \\
& \geq \delta G(\tau, s)+\frac{6 \delta}{5}(1-\tau) \int_{0}^{1} G(s, v) v d v \\
& \geq \delta H(\tau, s), \forall \tau, s \in[0,1] .
\end{aligned}
$$

Let $Q$ and $K$ denote two cones such that: Let the set

$$
Q=\{u \in C(J, E), u(t)=\geq \theta, t \in J\}
$$


and

$$
K=\left\{u \in Q, u(t) \geq \delta u(s), t \in J_{\delta}, s \in[0,1]\right\} .
$$

It is easy to see that $K$ is a cone of $C(J, E)$. We will make use of the following Lemma:

Lemma 3.7. Suppose that $(H)$ holds and $L_{l}<\left(B_{H}\right)^{-1}$. Then for each $l>0, T$ is a strict-set contraction on $Q \cap B_{l}$, that is there exists a constant $\gamma_{l}=B_{H} L_{l}$, such that

$$
\psi(T(S)) \leq \gamma_{l} \psi(S), \forall S \subset Q \cap B_{l},
$$

where $B_{l}=\left\{u \in C(J, E),\|u\|_{c} \leq l\right\}$.

Proof. $\quad$ By $(H), f$ is uniformly continuous on $J \times\left(P \cap S_{l}\right)$. From Lemma 2.16 , we obtain

$$
\psi(f(J \times S))=\max _{t \in J} \psi\left(f(t, S) \leq L_{l} \psi(S) .\right.
$$

Since $f$ is uniformly continuous and bounded on $S \subset Q \cap B_{l}$, then $T$ is continuous and bounded from $Q \cap B_{l}$ into $Q$.

Lemma 3.8. Suppose that $(H)$ holds. Then $T(K) \subset K$ and $T: K \longrightarrow K$ is a strict-set contraction.

Proof. We have from Lemma 3.6 that

$$
\begin{aligned}
\min _{t \in J_{\delta}} T u(t) & =\min _{t \in J_{\delta}} \int_{0}^{1} H(t, s) f(s, u(s)) d s \\
& \geq \delta \int_{0}^{1} H(\tau, s) f(s, u(s)) d s \\
& =T u(\tau), \forall \tau \in J .
\end{aligned}
$$

Therefore, $T u \in K$ and $T(K) \subset K$.

Furthermore, we have

$$
\begin{aligned}
\psi(T(S(t))) & =\psi\left(\int_{0}^{1} H(t, \tau) f(\tau, S(\tau)) d \tau\right) \\
& \leq \psi\left(\int_{0}^{1} \frac{2}{5} f(\tau, S(\tau)) d \tau\right)
\end{aligned}
$$




$$
\begin{aligned}
& \leq \frac{2}{5} \psi\left(\int_{0}^{1} f(\tau, S(\tau)) d \tau\right) \\
& \leq \frac{2}{5} \psi\left(\int_{0}^{1} \max _{t \in J} f(\tau, S(\tau)) d \tau\right) \\
& =\frac{2}{5} \psi(f(J, S)) \\
& \leq \frac{2}{5} L_{l} \psi(S) \\
& =\gamma_{l} \psi(S) .
\end{aligned}
$$

This prove that $T: K \longrightarrow K$ is a strict-set contraction.

We also consider the associated linear eigenvalue problem

$$
\begin{cases}u^{\prime \prime}(t)+u(t)=\mu u(t) & 0<t<1 \\ u(0)=\int_{0}^{1} s u(s) d s=u(1)=\theta, & \end{cases}
$$

Lemma 3.9. The linear eigenvalue problem (3.15) has a unique positive eigenvalue $\mu_{\star}>0$.

Proof. Let the set

$$
X=\left\{u \in C^{1}(J, E), u(0)=\int_{0}^{1} s u(s) d s u(1)=\theta\right\}
$$

be equipped with the norm defined for $u \in X$ by $\|u\|_{X}=\sup _{t \in J}\left\|u^{\prime}(t)\right\|$ and $K_{X}=K \cap X$ isaconeonX.

Considertheoperator $\mathrm{L}: \mathrm{X} \rightarrow X$ defined for $u \in X$ by

$$
L u(x)=\int_{0}^{1} H(t, s) u(s) d s,
$$

where $H$ is the green's function defined in (3.13).

Clearly that $\mu_{\star}>0$ is a positive eigenvalue of (3.15) if and only if $\mu_{\star}^{-1}>0$ is a positive eigenvalue of $L$. In view of Lemma 2.15, let us prove that $L\left(K_{X}^{\star}\right) \subset \operatorname{int}\left(K_{X}\right)$. To this end, consider the set

$$
O=\left\{u \in K_{X}, u>\theta \text { in }(0,1), u^{\prime}(0)>\theta, u^{\prime}(1)<\theta\right\} .
$$


We have $O \subset K_{X}$ and the complement of a set $O_{X}$ is $O^{c}$, such that

$$
O^{c}=F_{1} \cup F_{2} \cup F_{3},
$$

where

$$
\begin{gathered}
F_{1}=\left\{u \in X, \exists x_{0} \in[0,1] \text { with } u\left(x_{0}\right) \leq \theta\right\}, \\
F_{2}=\left\{u \in X, u^{\prime}(0) \leq \theta\right\}, \\
F_{3}=\left\{u \in X, u^{\prime}(1) \geq \theta\right\} .
\end{gathered}
$$

In the fact $F_{1}, F_{2}$ and $F_{3}$ are closed sets on X. To this aim let $\left(u_{n}\right)_{n} \subset$ $F_{1}$, tending to $u$ in $X$ and $\left(x_{n}\right)_{n} \subset[0,1]$ tending to $\bar{x}$ in $[0,1]$, with $u^{\prime}\left(x_{n}\right) \leq \theta$. We distinguish the following cases

- $\bar{x} \in] 0,1\left[\right.$; in such situation $u(\bar{x})=\lim _{n \rightarrow+\infty} u_{n}\left(x_{n}\right) \leq \theta$ and $u \in F_{1}$.

$\bullet \bar{x}=0$; in this case we obtain

$$
u^{\prime}(0)=\lim _{n \rightarrow+\infty} \frac{u_{n}\left(x_{n}\right)}{x_{n}} \leq \theta, \text { and } u \in F_{2} .
$$

- $\bar{x}=1$; in this case we obtain

$$
u^{\prime}(1)=\lim _{n \rightarrow+\infty} \frac{u_{n}^{\prime}\left(x_{n}\right)}{x_{n}-1} \geq \theta \text { and } u \in F_{3} .
$$

Therefore $O$ is an open subset of X.

On the other hand, let $u \in K_{X} \backslash\{\theta\}$ and $v=L u$, clearly $v>\theta$ on $(0,1)$, and we have

$v^{\prime}(t)=\int_{0}^{1} \frac{\partial G}{\partial t}(t, s) u(s) d s=\int_{0}^{1}\left(\frac{s^{3}}{5}-\frac{6}{5} s\right) u(s) d s+\int_{t}^{1} u(s) d s, \forall t \in[0,1]$.

Then

1. $v^{\prime}(0)=\int_{0}^{1}\left(\frac{s^{3}}{5}-\frac{6}{5} s+1\right) u(s) d s>\theta$

2. $v^{\prime}(1)=\int_{0}^{1}\left(\frac{s^{3}}{5}-\frac{6}{5} s\right) u(s) d s<\theta$.

That is,

$$
L\left(K_{X}^{\star}\right) \subset O \subset \operatorname{int}\left(K_{X}\right)
$$


Finally, lemma 2.15 guarantees existence of a unique positive eigenvalue of $L$ and we have $\mu_{\star}^{-1}=\theta_{K_{X}}$.

Let introduce the following notations

$$
f^{0}=\limsup _{u \rightarrow 0}\left(\max _{0 \leq t \leq 1} \frac{f(t, u)}{u}\right) \quad f^{\infty}=\limsup _{u \rightarrow \infty}\left(\max _{0 \leq t \leq 1} \frac{f(t, u)}{u}\right)
$$

Theorem 3.10. The problem (3.11) admits a positive solution whenever one of the following conditions:

$$
f^{\infty}<\mu_{\star}<f^{0}
$$

Proof. Let $L: E \longrightarrow E$ be the operator defined by (3.16). It is easy to see that $L$ is an increasing and strict set contraction operator.

Since $L(K) \subset K_{X}$, it follows from iv) Remark 2.13 that

$$
\mu_{\star}^{-1}=\theta_{K}^{L}
$$

where $K_{X}$ is the cone defined in the proof of Lemma 3.9.

Let $F: K \rightarrow K$, the Nemytskii operator defined for $u \in K$ by $F u(x)=$ $f(x, u(x))$. We present the proof of Theorem 3.10 in the case where (3.17) holds. Hypothesis (3.17) implies that there exists $\epsilon>0$, small enough such that

$$
\left(\mu_{\star}+\epsilon\right) u-H u \leq F u \leq\left(\mu_{\star}-\epsilon\right) u+c \text { for all } u \in K
$$

where $H u(x)=\max \left\{f(x, u(x))-f^{0} u(x), 0\right\}$. So, we get:

$$
\alpha L u-L H u \leq T u \leq \beta L u+B_{H} c \text { for all } u \in K,
$$

where $B_{H}=\frac{2}{5}, \alpha=\left(\mu_{\star}+\epsilon\right)$ and $\beta=\left(\mu_{\star}-\epsilon\right)$.

We introduce the following notation:

$$
h(u)=\alpha L u-L H u, g(u)=\beta L u+c M
$$

So we have

$$
h(u) \leq T u \leq g(u) \text { for all } u \in K
$$

Using the fact that $H(u)=\circ(\|u\|)$ near 0 , we may show that $h^{\prime}(0)$ is the derivative of $h$ along $K$ at zero, and $g^{\prime}(0)$ is the derivative of $g$ along $K$ at $\infty$ such that

$$
\frac{1}{\beta} g^{\prime}(\infty)[u]=\frac{1}{\alpha} h^{\prime}(0)[u]=L u, \forall u \in K .
$$


With this aim, let $u \in K$, there exists $\omega>0$ such that

$$
\left\|h(u)-h^{\prime}(0)[u]\right\|=\|L H u\| \leq \omega\|H u\| .
$$

Therefore, we have:

$$
\lim _{u \in K,\|u\| \longrightarrow 0} \frac{\left\|h(u)-h^{\prime}(\infty)[u]\right\|}{\|u\|} \leq \omega \lim _{\|u\| \longrightarrow 0} \frac{\|H u\|}{\|u\|} .
$$

This means that

$$
\lim _{u \in K,\|u\| \longrightarrow 0} \frac{\left\|h(u)-h^{\prime}(0)[u]\right\|}{\|u\|}=0 .
$$

Therefore, we have:

$$
\lim _{u \in K,\|u\| \longrightarrow+\infty} \frac{\left\|g(u)-g^{\prime}(\infty)[u]\right\|}{\|u\|}=\lim _{\|u\| \longrightarrow+\infty} \frac{c M}{\|u\|} .
$$

Clearly that $h^{\prime}(0), g^{\prime}(\infty) \in C_{K}(E)$ and $\theta_{P}^{h^{\prime}(0)}=\alpha \mu_{\star}^{-1}$ and $r\left(g^{\prime}(\infty)\right)=$ $\beta \mu_{\star}^{-1}$. So from the choice of $\alpha$, and $\beta$, we have

$$
r\left(g^{\prime}(\infty)\right)<1<\theta_{P}^{h^{\prime}(0)} .
$$

We deduce from the Theorem 3.3 existence results for positive solution to the bvp (3.11).

Acknowledgement. The author would like to thank his laboratory, Fixed Point Theory and Applications, for supporting this work.

\section{References}

[1] H. Amann, "Fixed point equations and nonlinear eigenvalue problems in ordered Banach spaces”, SIAM Review, vol. 18, no. 4, pp. 620-709, 1976.

[2] H. Amann, "Fixed points of asymptotically linear maps in ordered Banach spaces", Journal of functional analysis, vol. 14, no. 2, pp. 162-171, 1973.

[3] A. Benmezai and B. Boucheneb, "A fixed point theorem for positive strict set-contractions mappings and its application to Urysohn type integral equations", Archiv der mathematik, vol. 105, pp. 389-399, 2015. 
[4] A. Benmezai, John R. Graef, and L. Kong, "Positive solutions for the abstract Hammerstein equations and applications", Communications in mathematical analysis, vol. 16, no. 1, pp. 47-65, 2014.

[5] A. Benmezai and S. Mechrouk, "Positive solutions for the nonlinear abstract Hammerstein equation and application to $\phi$-Laplacian BVPs", Nonlinear differential equations and applications, vol. 20, pp. 489-510, 2013.

[6] K. Deimling, Nonlinear functional analysis. Berlin: Springer, 1985.

[7] D. Guo, Y. J. Cho, and J. Zhu, Partial ordering methods in nonlinear problems. New York, NY: Nova Science, 2004.

[8] D. Guo and V. Lakshmikantham, Nonlinear problems in abstract cones. Boston, MA: Academic Press, 1988.

[9] D. Guo and V. Lakshmikantham, "Multiple solutions of two-point boundary value problem of ordinary differential equations in Banach space", Journal of mathematical analysis and applications, vol. 129, no. 1, pp. 211-222, 1988.

[10] D. Guo, V. Lakshmikantham, and X. Liu, Nonlinear integral equations in abstract spaces. Dordrecht: Kluwer, 1996.

[11] M. A. Krasnosel'Skii and L. F. Boron, Positive solutions of operator equations. Groningen: P. Noordhoff, 1964.

[12] S. Mechrouk, "Fixed point theorems in cones and application", Mediterranean journal of mathematics, vol. 17, no. 2, Art. ID. 59, 2020.

[13] R. D. Nussbaum, "The fixed point index for local condensing maps", Annali di matematica pura ed applicata, vol. 89, pp. 217-258, 1971.

[14] E. Zeidler, Nonlinear functional analysis and its applications, vol. 1. New York, NY: Springer, 1986.

\section{Salima Mechrouk}

Faculty of Sciences,

Université de Boumerdes, U.M.B.B.,

Boumerdes,

Algeria

e-mail: mechrouk@gmail.com

e-mail: mechrouk@univ-boumerdes.dz 